\title{
Commentary on Operationalizing Partnership Between a Traditional Research Institution and a Massage School
}

\author{
Erika Larson, LMT, MSBIOE, BSME \\ Atlanta School of Massage, Atlanta, GA, USA
}

\section{INTRODUCTION}

In the field of therapeutic massage and bodywork (TMB) there is a lack of TMB programs at traditional research institutions (TRIs). As a result, TMB research may align with the expertise and interests of researchers at TRIs, yielding outcomes with limited clinical utility for TMB practitioners. ${ }^{(1,2)}$ Creating pathways for practitioners to become researchers and establishing TMB programs at TRIs both require time and monetary investment. An achievable shorter-term goal is establishing collaborative partnership between a TRI and a TMB program. Such partnership may be necessary to build research capacity in the TMB field. $(2,3,4)$

In a partnership between a TRI and a TMB program, the TMB program serves as a subject-matter expert. The purpose of this commentary is to describe the process of the capacity building for the Atlanta School of Massage (ASM) becoming a research and implementation partner for a National Institute of Health (NIH)-funded study with Emory University's School of Medicine (Emory).

\section{The Project}

In 2011 Mark Rapaport, MD, moved from Cedars-Sinai Medical Center (Cedars-Sinai) in Los Angeles, CA, to Emory; with him came a NIHfunded project, Massage for Generalized Anxiety Disorder (MGAD) ${ }^{(5)}$ (ClinicalTrials.gov identifier: NCT01337713). To conduct this project in Atlanta, GA, Emory identified ASM as a research partner. MGAD utilized two types of TMB interventions. (5) As ASM had limited research experience, Emory guided the process by providing support and research infrastructure.

\section{Framework for Operationalizing Partnership}

Collaborative partnerships have been studied across disciplines. ${ }^{(6,7)}$ Garland and BrookmanFrazee $^{(6)}$ refined a model developed for a specific type of collaborative partnerships - research-community practice partnerships. This model is comprised of three phases: (a) partnership formation; (b) execution; (c) sustainability. ${ }^{(6,8)}$ The partnership formation phase provides structure to describe operationalization of the Emory-ASM partnership. During partnership formation, both interpersonal processes and operational processes are addressed to create a functional partnership. ${ }^{(6,8)}$

\section{INTERPERSONAL PROCESSES}

Interpersonal processes necessary for collaborative partnership operationalization include: (a) complementary goals; (b) establishing relationships and trust; and (c) roles $\&$ responsibilities. ${ }^{(6)}$

\section{Complementary Goals}

Stakeholder goals need to be complementary and further the collaboration. ${ }^{(6,7)}$ ASM hoped to build its research capacity to progress toward a goal of conducting grant-funded research at ASM. Emory sought partnership with ASM out of need for qualified methodologic experts in TMB. To realize Emory's goal, an appropriately-sized team of massage therapists capable of fulfilling the specialized requirements of the study was assembled. To that end, steps for identifying, recruiting, and hiring research massage therapists (rMTs) are described.

\section{Application and hiring process for rMTs}

In Fall 2011, ASM initiated rMT recruitment efforts to support the transition of MGAD to Emory. The ASM research liaison based hire requirements on those established by the Cedars-Sinai team, which aligned with hire requirements specified in other studies with TMB interventions. ${ }^{(9,10,11)}$ Based on the anticipated subject load, three rMTs would be recruited. Hire requirements included agreement to perform essential job duties, educational requirements, and licensure/certification requirements. Essential job duties included, but were not limited to, (a) providing the intervention protocol to study subjects; (b) working with other research personnel, including the research coordinator; and (c) ensuring compliance and maintaining proper documentation as mandated by the standard operating protocols (SOPs) approved 
by the Institutional Review Board (IRB). Applicants who had completed two or more years of college were preferred. Candidates had to have completed an accredited program in massage therapy and hold a current state massage license. ${ }^{(12)}$ Hire requirements were modified to reflect the new study location, as laws governing TMB practice vary throughout the United States. ${ }^{(12,13,14)}$

The ASM research liaison contacted individuals he thought would be qualified and interested in participating. Additionally, one massage therapist contacted the ASM research liaison and the principal investigator $(\mathrm{PI})$, inquiring about research opportunities after attending a community presentation given by the PI. Selected applicants submitted documentation, applied for the position, and completed a two-part interview process (September 2011 to November 2011) that included an interview with the ASM research liaison and a hands-on technique assessment involving a massage to the ASM research liaison. ASM offered rMT positions to three applicants who completed ASM-specific paperwork in December 2011. Each rMT had graduated from ASM in the past five years (Table 1). The hiring process concluded with rMTs completing research requirements (Collaborative Institutional Training Initiative (CITI) certification and IRB approval). The rMTs were required to complete CITI certification prior to the January research personnel training.

\section{Establishing Relationships and Trust}

In the Emory-ASM collaboration, establishment of relationships and trust began in a face-to-face initial team meeting and research training that ASM volunteered to host. Together, Emory and the ASM research liaison drafted an agenda for the initial team meeting. The scientific research administrator e-mailed meeting invitations and provided rMTs with the MGAD grant, MGAD SOPs, and related journal articles. ${ }^{(15,16)}$ Arrangements were made for a Cedars-Sinai rMT provide intervention protocol training and review research conduct expectations. Besides MGAD, this Cedars-Sinai rMT had participated in two other studies led by the PI. ${ }^{(15,16)}$

The initial research personnel meeting on January 16,2012 , aimed to establish rapport, elucidate guiding

TABle 1. Demographics of Study Research Massage Therapists

\begin{tabular}{lcccc}
\hline rMT & Gender & $\begin{array}{c}\text { Professional } \\
\text { Experience }^{a}\end{array}$ & $\begin{array}{c}\text { Massage } \\
\text { School }\end{array}$ & $\begin{array}{c}\text { Research } \\
\text { Experience }\end{array}$ \\
\hline 1 & female & 1.6 years & ASM & no \\
2 & male & 3.1 years & ASM & no \\
3 & female & 4.9 years & ASM & yes \\
\hline
\end{tabular}

aprofessional experience defined as time elapsed from state licensure $^{(12)}$ to the initial research personnel meeting. practices in research, define roles, review SOPs (including a visit overview), and train rMTs on both intervention protocols. The MGAD study team consisted of the PI, scientific research administrator, physicians, biostatistician, research coordinator, research assistants, ASM research liaison, and rMTs (Figure 1). All, except the study physicians and biostatistician, attended the initial meeting. To build rapport by establishing commonalities, the PI initially engaged the research team in casual conversation by inquiring of each individual their ideal music during a massage. The structure of the initial meeting illustrated that all research personnel are vital to the success of the study. Sections of the meeting were led by the team member with expertise in that domain. The PI provided context and rationale for the study and emphasized the need for intra- and inter-site consistency assessed through ongoing quality control measures. The Cedars-Sinai rMT talked everyone through the experimental protocol and rMT responsibilities; demonstrated both the Swedish massage therapy and light touch intervention protocols; and checked for consistency in technique amongst rMTs. The "subjects" during the intervention protocol demonstrations and rMT-practice of the interventions were other MGAD research personnel, including the research coordinator and the scientific research administrator. MGAD initial training was similar to initial training for rMT-participation in another investigation. ${ }^{(11)}$

Previous research suggests that both interventions may have clinical benefit. ${ }^{(16)}$ Light touch was considered an active control during MGAD. Research personnel were repeatedly encouraged to share and collaborate. In particular, the Cedars-Sinai rMT emphasized the importance of rMTs working together as the experts on the TMB interventions. All research personnel contribute to the validity of the data and open communication is important to ensuring subject safety and study integrity. Efforts were made to foster use of a common language.

Building on the collaborative atmosphere established at the initial research personnel meeting, the scientific research administrator scheduled weekly meetings starting January 18, 2012, to discuss SOPs. During SOP meetings, current TMB research was discussed. The PI sent links to scientific papers relevant to TMB research to rMTs to support improving rMT research literacy. The lead rMT shared resources with other rMTs to promote research literacy including research-related continuing education options, NIH video library lectures, and relevant textbooks. As the study start approached, SOP meetings rolled into weekly research personnel meetings.

\section{Roles and Responsibilities}

Defining roles and expected contributions, and determining distribution of power during the partnership formation phase are essential in a collaborative 


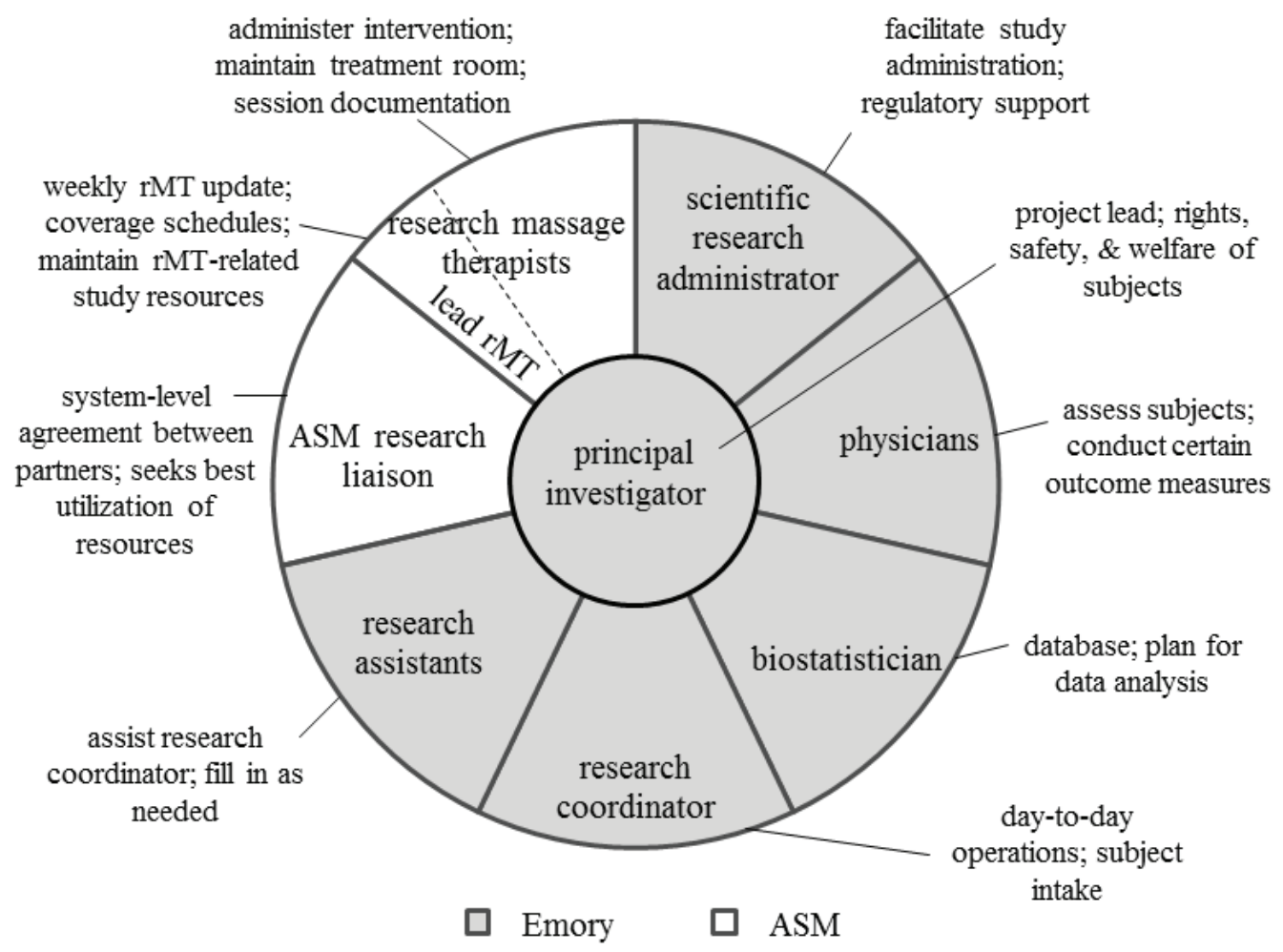

FIGURE 1. MGAD personnel and primary responsibilities. Principal investigator also served as a MGAD study physician.

partnership. ${ }^{(6,7)}$ Partnership success is a shared burden amongst all stakeholders. In addition to establishing rapport, the initial research personnel meeting defined a vision for the rMTs: to document the training received that day in a manner that would be repeatable, executable, and teachable. To carry out this vision, rMTs met weekly to clarify existing Cedars-Sinai resources and create additional resources. Three resources were deemed essential to finalize prior to commencing active treatment: (a) rMT script; (b) intervention protocols; and (c) intake convey. The ASM research liaison assigned each rMT one task.

\section{rMT script}

Script usage was intended to standardize interactions between the rMT and the subject, prompt the rMT to relay required information to the research coordinator, and provide information on visit flow. The rMT script was based on MGAD SOPs and information provided by the Cedars-Sinai rMT at the initial research personnel meeting. The script specified where the rMT should be and an overview of visit flow.

\section{Intervention protocols}

The intervention protocols specify contact type, directionality, and progression for a standardized 45-minute treatment. Drawing from the initial research personnel meeting, descriptions in the MGAD grant, previously published journal articles from the PI, ${ }^{(15,16)}$ and the Cedars-Sinai intake convey, intervention protocol refinement was completed in stages to track changes and allow for feedback from research personnel.

\section{Intake convey}

The intake convey is a specific, but succinct, summary of each intervention protocol in layman's terms intended to communicate what to expect during treatment to each subject. The Emory MGAD intake convey was based on the detailed Cedars-Sinai intake convey and the intervention protocols. This deliverable was utilized in subject credibility/expectancy measures during MGAD. ${ }^{(5)}$

Subject recruitment began in earnest after rMT script, intervention protocols, and intake convey completion. While awaiting subject enrollment, rMTs had an opportunity to practice the protocol on research assistants, comparable to mock sessions utilized in another TMB study. (11) During the visit, the "subject" would ask questions or attempt to distract the rMT. Each rMT worked on the same two "subjects." Feedback forms from each session were discussed at a weekly research personnel meeting, leading to further protocol refinement to ensure intra-rMT and inter-rMT consistency. Mock sessions were an effective training exercise that bolstered rMT confidence in their role of providing standardized TMB interventions in a research setting. 
A walkthrough of facilities and procedures was completed to confirm readiness for MGAD research personnel. Solutions for identified issues from the walkthrough were discussed and implemented. Aspects of study coordination were reviewed. If an rMT was unable to see a subject due to an existing relationship or having to miss a session, the subject would be rescheduled or another rMT would be called.

\section{Role of rMT}

The role of rMT was defined to include: (a) maintenance of protocol integrity; (b) minimization of variations from documented practices and procedures; and (c) thorough documentation of unavoidable variations. Acknowledging that the role of rMT in MGAD was a departure from community massage practice involving manualized intervention protocols, a scientist-generated treatment notes page, no intake interview, and no home care, the ASM research liaison developed a training presentation which included a list of key differences between the type of research TMB MGAD utilized and community massage practice (Table 2). More thorough descriptions of community TMB practice have been previously published. ${ }^{(9,14,17)}$ The supportive team environment eased rMT transition from community massage practice to research massage therapy.

Finalized MGAD rMT expected contributions included: (a) maintenance of the treatment room; (b) provision of the intervention protocol; (c) session documentation, including report of all discrepancies/ deviations; (d) preparation of a weekly rMT update; and (e) participation in weekly research personnel meetings (Figure 1).

Table 2. Research Massage Therapy vs. Community Massage Practice

\begin{tabular}{lcc}
\hline & $\begin{array}{c}\text { Research Massage } \\
\text { Therapy }\end{array}$ & $\begin{array}{c}\text { Community Massage } \\
\text { Practice }\end{array}$ \\
\hline Provider & $\begin{array}{c}\text { research massage } \\
\text { therapist } \\
\text { study subject }\end{array}$ & massage therapist \\
$\begin{array}{l}\text { Recipient of } \\
\text { Treatment }\end{array}$ & massage client \\
Type of Treatment & $\begin{array}{c}\text { standardized } \\
\text { intervention } \\
\text { standardized }\end{array}$ & $\begin{array}{c}\text { individualized } \\
\text { treatment }\end{array}$ \\
$\begin{array}{l}\text { Session Length } \\
\text { Boundary } \\
\text { Negotiation }\end{array}$ & $\begin{array}{c}\text { varies } \\
\text { individuals unwilling } \\
\text { to receive the } \\
\text { entire protocol } \\
\text { are not chosen for } \\
\text { participation }\end{array}$ & $\begin{array}{c}\text { ongoing and } \\
\text { adaptive }\end{array}$ \\
$\begin{array}{l}\text { Provider-Recipient } \\
\text { Relationship }\end{array}$ & $\begin{array}{c}\text { mediated by script } \\
\text { and research } \\
\text { coordinator; constant } \\
\text { over time }\end{array}$ & $\begin{array}{c}\text { interpersonal; built } \\
\text { over time }\end{array}$ \\
& & \\
\hline
\end{tabular}

\section{OPERATIONAL PROCESSES}

The operational processes of leadership, funding resources \& administrative support, and communication methods are inter-related processes. Operational processes managing Cedars-Sinai MGAD were adapted for the Emory-ASM partnership.

\section{Leadership}

Leadership of a collaborative partnership between a TRI and a massage therapy school requires special skill. Leaders must see the big picture, maintain a balance of power, monitor rapport, sustain team morale, and maintain level of involvement amongst stakeholder groups. ${ }^{(6,7)}$ The PI for the Emory-ASM collaboration is a charismatic leader with experience working with massage therapists. ${ }^{(3,15,16)}$

The operational process of leadership also includes leadership within the project. ${ }^{(6)}$ Sustainability of a collaborative partnership involves matching governance duties with skills and resources ${ }^{(6,7)}$ — the partnership's success depends on all involved. Emory led the MGAD workflow setup and subject recruitment. The responsibility of developing and refining documents forming the basis for the intervention (protocol, intake convey, script) fell to the rMTs. These documents guided rMT conduct within the partnership, which required different skill sets from operating as a community massage practitioner.

An unofficial chain of command arose during the formation phase: rMTs, lead rMT, ASM research liaison, research coordinator, scientific research administrator, and PI. Within the team, workgroups took on specific tasks. Progress was reported at weekly research personnel meetings.

\section{Funding, Resources, and Administrative Support}

MGAD was grant-funded. The grant included provision of a research coordinator. Emory also contributed the scientific research administrator, the PI, and research infrastructure (such as IRB) (Figure 1 ). This arrangement may skew the balance of power toward TRI, ${ }^{(2,6)}$ but enabled ASM to engage in this level of grant-funded research.

The scientific research administrator and the ASM research liaison had meetings throughout partnership formation. Meetings included discussions of specific questions (e.g., what lubricant would be utilized during the massage intervention) and broader points (e.g., the role of the rMT). Quality control and methods for its ongoing assessment were discussed. In addition, the financial aspect of the partnership was explored. The type of financial agreement (contract, service agreement, or other), pay rate, and the duties for which rMTs would be compensated were addressed. Of note, rMTs were 
hired with neither a clear idea of what responsibilities would fall under the purview of rMT duties nor a defined pay rate.

ASM incurred financial burden and resource allocation burden by participating in the study. To provide qualified rMTs for MGAD, ASM paid for rMT CITI training completion. The responsibilities of the ASM research liaison role (Figure 1) were added to the job duties of an existing staff member. The majority of MGAD-related activities would take place at Emory, so the logistics of documenting hours worked had to be considered.

Stakeholders provide the majority of resources in a collaborative partnership. ${ }^{(7)}$ The rMTs were one of the resources developed for the Emory-ASM partnership.

\section{Communication Methods}

Many communication methods utilized in the Emory-ASM partnership formation for MGAD served to satisfy IRB requirements. Face-to-face meetings are important for establishing trust, especially during operationalization. ${ }^{(6)}$ The initial team development meeting and subsequent SOP meetings took place in person. After transitioning to mandatory weekly research personnel meetings, research personnel were given the option to call into meetings. At weekly research personnel meetings all aspects of MGAD were discussed, including any questions, rMT performance, and suggestions for improvement. Meeting reminders and pertinent time-sensitive information between meetings were e-mailed.

The lead rMT e-mailed rMT coverage schedules monthly, which listed the hours of availability of the primary rMT on a given day and a secondary rMT whenever possible. The research coordinator and rMTs achieved acceptable compromise between the desired coverage hours to support MGAD and rMT availability. All MGAD rMTs had primary employment outside the study. The research coordinator e-mailed a subject schedule weekly and called rMTs with last minute scheduling requests.

To develop and refine resources for MGAD, rMTs met in person and collaborated via an online shared drive. For MGAD participation, each rMT had two email addresses: an ASM e-mail address and an Emory e-mail address. The ASM e-mail address facilitated access to ASM's online shared drive, where rMTs kept a repository of study-related information. To work on MGAD at Emory, rMTs had to have a sponsored Emory account. This account was managed through an Emory e-mail address. In addition, IRB approval and CITI completion were documented under the Emory e-mail address identifier.

Supplementary documents were developed by rMTs to facilitate communication, address emerging needs, and thoroughly document rMT duties. These supplementary documents included: (a) Swedish massage vocabulary; (b) working study vocabulary for rMTs; (c) draping guide; and (d) room \& table preparation guide.

\section{DISCUSSION \& DEVELOPMENT}

This collaborative partnership was formed around an ongoing study, with a clearly defined task of minimizing variations with minimal elapsed between active status at each site. However, challenges were still encountered. With only one afternoon of handson training with the Cedars-Sinai protocol expert, rMTs had to rely on notes while refining resources; a video of the intervention protocol would have been helpful. ${ }^{(18)}$

The three rMTs hired for the study had similar training, which minimized certain barriers, such as having consistent terminology for describing TMB. A barrier in TMB research is the vast array of interpretations of techniques. ${ }^{(1,18)}$ Terminology differences between Cedars-Sinai and Emory sites observed during initial training prompted rMTs from ASM to define TMB terminology utilized for MGAD and disseminate amongst the research personnel. Shared language is vital to partnership functionality. $(6,7,8,18)$ While the rMTs had similar foundational educational experiences, their continuing education experiences varied. In TMB practice, scope of practice depends on training and location. ${ }^{(14,17)}$ Factors which may influence interpretation of TMB study results include types of therapies practiced, ${ }^{(17)}$ training duration, ${ }^{(19)}$ and experience. ${ }^{(17)}$

Traditional structure for TMB efficacy trials may limit generalizability. ${ }^{(1,9,17)}$ However, there may be merit to testing a TMB protocol as the practice of medicine is based in protocol. MGAD design reflects previous studies conducted by the PI, $(3,15,16)$ thereby creating continuity. The research coordinator performed intake for all MGAD subjects and relayed pertinent information to the rMTs. Use of a standardized protocol allowed the study team to establish contraindications.

Emory guided ASM through protections governing human-subject research with the goal of developing a long-term research partner. The scientific research administrator requested facilitated rMT setup in IRB by obtaining a sponsored Emory account for each rMT. Research personnel collectively formed solutions to ensure SOPs met IRB requirements. Handwritten rMT treatment notes had to be input into the MGAD database after each treatment session. MGAD rMTs proposed entering notes verbatim in the database after each session. ASM stakeholders willingly complied with requirements as participating in this partnership built capacity, thereby helping progress toward achieving the goal of conducting on-site, grant-funded research.

During operationalization, perspective and expertise for each research personnel member was valued. 
Successful partnership requires openness on both sides to learning from each other. To sustain this collaborative partnership, the massage school may need to examine methods for improving research literacy and increasing research capacity, both as an organization and within curricula, to better articulate TMB priorities. The TRI could then adapt outcomes and reporting practices to reflect these priorities.

\section{Proximal Outcomes}

Efforts made managing the interpersonal processes and operational processes during the partnership formation phase lay the groundwork for an effective partnership. The Emory-ASM collaborative partnership exhibited proximal outcomes associated with success: (a) partnership synergy; (b) knowledge creation and exchange; and (c) tangible products. ${ }^{(6,8)}$

Improved understanding and mutual respect amongst stakeholders were observed. Tangible products resulting from the MGAD Emory-ASM collaboration included MGAD completion and rMT-developed resources. The collaborative environment established during MGAD operationalization empowered rMTs to identify needs and address them. As the study progressed, rMTs added to the repository of accessible resources by developing: (a) unanticipated in-session events; (b) new rMT orientation to the facilities; (c) new rMT onboarding manual; (d) intervention protocol video; (e) abbreviated rMT script; and (f) rMT hire requirements. Through participation in MGAD operationalization and development of these supplementary materials, rMTs grasped and articulated the scientific vision of the study. Retraining sessions were proposed by rMTs as part of the ongoing monitoring and assessment procedures. ${ }^{(5)}$

\section{Conclusion and Sustainability}

This detailed commentary on the partnership formation process may be of benefit to others embarking on such a collaborative endeavor. In the past, we have published details how psychiatrists can collaborate with community massage therapists for optimal patient care. ${ }^{(20)}$ The example of MGAD operationalization here illustrates an additional path for allopathic practitioners to collaborate with TMB practitioners. Participating in research with a manualized protocol requires the specialized skill set of a rMT, and creation of a TMB research protocol involves more than developing standardized terminology. ${ }^{(1,18)}$ The success of this collaborative partnership illustrates a potential need to create a rMT career path. ${ }^{(2,3,4)}$ Involving rMTs in additional facets of the research process may yield results with greater relevance for TMB practice and consumers. (2) However an expanded rMT role may necessitate additional research training.
The phases and processes of the model Garland and Brookman-Frazee ${ }^{(6)}$ refined for effective collaborative partnerships are intended to inform each other and be revisited. Resources developed for MGAD, including the rMTs, have been useful in other Emory-ASM endeavors. ${ }^{(20,21)}$ Operationalizing an active study prepared the team for a subsequent project-conducting a study from its inception. ${ }^{(21)}$ Research personnel examined the partnership and refined it to address the demands of the new study. Each Emory-ASM endeavor has allowed rMTs opportunity to increasingly influence study design. The Emory-ASM partnership hopes to conduct multisite studies $^{(3)}$ to continue building on the partnership's success. Weekly research personnel meetings continue, allowing research personnel to regularly evaluate the partnership and implement changes as needed. (6) Studying the collaborative partnership itself may further inform sustainability practices. ${ }^{(8)}$

\section{CONFLICT OF INTEREST NOTIFICATION}

The author declares there are no conflicts of interest.

\section{COPYRIGHT}

Published under the CreativeCommons AttributionNonCommercial-NoDerivs 3.0 License.

\section{REFERENCES}

1. Baskwill A. A commentary on the role of randomized controlled trials in massage therapy. Int $J$ Ther Massage Bodywork. 2017;10(4):13. Available from: http://ijtmb.org/index.php/ ijtmb/article/view/375. Accessed May 17, 2018.

2. Kania-Richmond A, Menard MB, Barberree B, Mohring M. "Dancing on the edge of research"-What is needed to build and sustain research capacity within the massage therapy profession? A formative evaluation. J Bodyw Mov Ther. 2017;21(2):274-283.

3. Kennedy AB. From skeptic to vital partner in massage therapy research: an interview with Mark Hyman Rapaport, MD [editorial]. Int J Ther Massage Bodywork. 2018;11(2):1. Available from: https://www.ncbi.nlm.nih.gov/pmc/articles/ PMC5988343/pdf/ijtmb-11-1.pdf. Accessed June 06, 2018.

4. Menard MB, Weeks J, Anderson B, Meeker W, Calabrese C, O'Bryon D, et al. Consensus recommendations to NCCIH from research faculty in a Transdisciplinary Academic Consortium for Complementary and Integrative Health and Medicine. $J$ Altern Complement Med. 2015;21(7):386-394.

5. Rapaport MH, Schettler P, Larson ER, Edwards SA, Dunlop BW, Rakofsky JJ, et al. Acute Swedish massage monotherapy successfully remediates symptoms of generalized anxiety disorder: a proof-of-concept, randomized controlled study. $J$ Clin Psychiatry. 2016;77(7):883-891.

6. Garland AF, Brookman-Frazee L. Therapists and researchers: advancing collaboration. Psychother Res. 2015;25(1):95-107. 
7. Lasker RD, Weiss ES, Miller R. Partnership synergy: a practical framework for studying and strengthening the collaborative advantage. Milbank Q. 2001;79(2):179-205.

8. Brookman-Frazee L, Stahmer A, Stadnick N, Chlebowski C, Herschell A, Garland AF. Characterizing the use of researchcommunity partnerships in studies of evidence-based interventions in children's community services. Admin Policy Ment Health. 2016;43(1):93-104.

9. Munk N, Stewart K, Love MM, Carter E, Elder WG. The intersection of massage practice and research: community massage therapists as research personnel on an NIH-funded effectiveness study. Int J Ther Massage Bodywork. 2014;7(2):10-19. https:// www.ncbi.nlm.nih.gov/pmc/articles/PMC4051806/.Accessed July 9, 2018.

10. Sherman KJ, Cook AJ, Wellman RD, Hawkes RJ, Kahn JR, Deyo RA, et al. Five-week outcomes from a dosing trial of therapeutic massage for chronic neck pain. Ann Fam Med. 2014;12(2):112120.

11. Cherkin DC, Sherman KJ, Kahn J, Erro JH, Deyo RA, Haneuse $\mathrm{SJ}$, et al. Effectiveness of focused structural massage and relaxation massage for chronic low back pain: protocol for a randomized controlled trial. Trials. 2009;10(1):96.

12. Georgia Secretary of State, Georgia Massage Therapy Board. Massage Therapy-Georgia Board of Massage Therapy. Atlanta, GA: The Board; 2018. Available from: http://sos.ga.gov/index. php/licensing/plb/33. Modified July 24, 2018. Accessed July 24, 2018.

13. California Massage Therapy Council [website]. Sacramento, CA: California Massage Therapy Council. Available from: http://www.camtc.org/. Modified July 23, 2018. Accessed July 23, 2018.

14. Kennedy AB, Cambron JA, Sharpe PA, Travillian RS, Saunders RP. Process for massage therapy practice and essential assessment. J Bodyw Mov Ther. 2016;20(3):484-496.
15. Rapaport $\mathrm{MH}$, Schettler P, Bresee C. A preliminary study of the effects of a single session of Swedish massage on hypothalamic-pituitary-adrenal and immune function in normal individuals. J Altern Complement Med. 2010;16(10):1079-1088.

16. Rapaport MH, Schettler P, Bresee C. A preliminary study of the effects of repeated massage on hypothalamic-pituitary-adrenal and immune function in healthy individuals: a study of mechanisms of action and dosage. The J Altern Complement Med. 2012;18(8):789-797.

17. Porcino AJ, Boon HS, Page SA, Verhoef MJ. Exploring the nature of therapeutic massage bodywork practice. Int $J$ Ther Massage Bodywork. 2013;6(1):15-24. Available from: http:// www.ijtmb.org/index.php/ijtmb/article/view/168. Accessed July 14, 2018.

18. Sherman KJ, Dixon MW, Thompson D, Cherkin DC. Development of a taxonomy to describe massage treatments for musculoskeletal pain. BMC Complement Altern Med. 2006;6(1):24.

19. Moraska A. Therapist education impacts the massage effect on postrace muscle recovery. Med Sci Sports Exerc. 2007;39(1):34-37.

20. Rapaport MH, Schettler PJ, Larson ER, Carroll D, Sharenko $\mathrm{M}$, Nettles J, et al. Massage therapy for psychiatric disorders. Focus. 2018;16(1):24-31.

21. Kinkead B, Schettler PJ, Larson ER, Carroll D, Sharenko $\mathrm{M}$, Nettles J, et al. Massage therapy decreases cancer-related fatigue: results from a randomized early phase trial. Cancer. 2018;124(3):546-554.

Corresponding author: Erika Larson, LMT, MSBIOE, BSME, Atlanta School of Massage, 2 Dunwoody Park S., Suite 101, Atlanta, GA 30388, USA E-mail: erikalarson@atlantaschoolofmassage.edu 